\title{
Surface Telerobotics: Development and Testing of a Crew Controlled Planetary Rover System
}

\author{
Maria G. Bualat ${ }^{1}$, Terrence Fong, Mark Allan, Xavier Bouyssounouse, Tamar Cohen, \\ Lorenzo Flückiger, Ravi Gogna, Linda Kobayashi, Yeon Jin Lee, Susan Y. Lee, \\ Chris Provencher, Ernest Smith, Vinh To, Hans Utz, and DW Wheeler \\ NASA Ames Research Center, Moffett Field, CA 94035 \\ Estrellina Pacis \\ Space and Naval Warfare Systems Center, San Diego, CA 92152 \\ Debra Schreckenghost and Tod Milam \\ TRACLabs, Inc., Webster, TX 77598 \\ and \\ David Mittman and R. Jay Torres \\ Jet Propulsion Laboratory, Pasadena, CA 91109
}

\begin{abstract}
During Summer 2013, we conducted a series of tests to examine how astronauts in the International Space Station (ISS) can remotely operate a planetary rover. The tests simulated portions of a proposed mission, in which an astronaut in lunar orbit remotely operates a planetary rover to deploy a radio telescope on the lunar farside. In this paper, we present the design, implementation, and preliminary test results.
\end{abstract}

\section{Introduction}

TN planning for future exploration missions, architecture and study teams have made numerous assumptions about how crew can be telepresent on a planetary surface by remotely operating surface robots from space (i.e. from a flight vehicle or deep space habitat). ${ }^{1,2,3,4,5}$ These assumptions include estimates of technology maturity, existing technology gaps, and operational risks. These assumptions, however, have not been grounded by experimental data. Moreover, to date, no crew-controlled surface telerobot has been fully tested in a high-fidelity manner.

To address these issues, we developed the "Surface Telerobotics" tests to do three things:

1) Demonstrate interactive crew control of a mobile surface telerobot in the presence of short communications delay.

2) Characterize a concept of operations for a single astronaut remotely operating a planetary rover with limited support from ground control.

3) Characterize system utilization and operator work-load for a single astronaut remotely operating a planetary rover with limited support from ground control.

\section{Proposed Lunar Waypoint Mission}

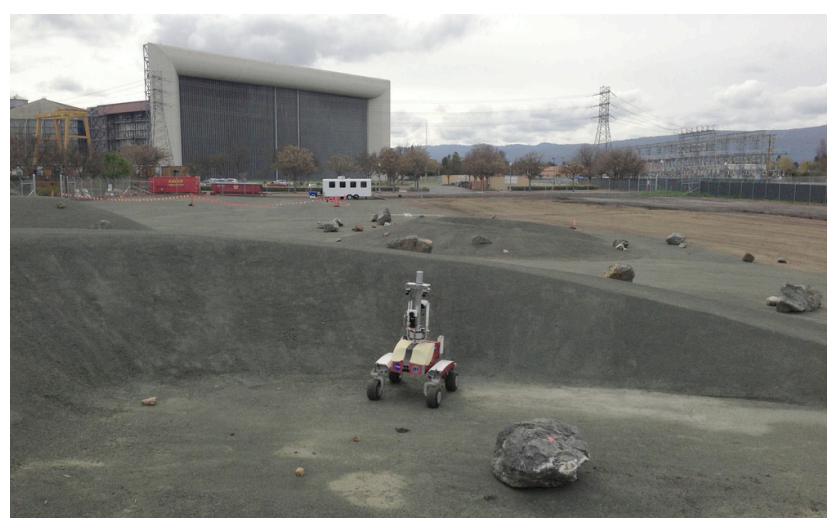

Figure 1. Lunar analog test site at the NASA Ames Research Center.
Surface Telerobotics focused on simulating a possible future human-robotic "Lunar Waypoint" mission. Exploration of the farside of the Moon is currently seen as a possible early goal for missions beyond low Earth orbit using the Orion Multi-Purpose Crew Vehicle (MPCV).

One leading concept, the "Orion MPCV L2Farside" mission, proposes to send a crewed MPCV to the L2 Earth-Moon Lagrange point, where the combined gravity of the Earth and Moon allows a spacecraft to easily maintain a stationary orbit over the lunar farside ${ }^{6}$. From L2, an astronaut would remotely operate a robot to perform high-priority surface science work, such as deploying a polyimide film-based radio telescope. Observations of the Universe's first

\footnotetext{
${ }^{1}$ Deputy Director, Intelligent Robotics Group, NASA Ames Research Center, M/S 269-3, Moffett Field, CA.
} 
stars/galaxies at low radio frequencies is a key science objective of the 2010 Astronomy and Astrophysics Decadal Survey. ${ }^{7}$ Such a mission would also help prepare for subsequent deep-space human exploration missions. For example, a similar strategy might be employed by humans to explore the surface of Mars from orbit ${ }^{5}$.

To study this human-robot exploration approach, Surface Telerobotics simulated four phases of the Orion MPCV L2-Farside mission concept: pre-mission planning, site survey, simulated telescope deployment, and inspection of deployed telescope. We performed these four phases in sequence. After pre-mission planning, we performed the other three phases during three test sessions with ISS crew. Each crew session included an hour of on-board crew training for the robot user interface, and two hours of mission operations.

\section{A. Pre-Mission Planning}

We performed the pre-mission planning phase in Spring 2013. A mission planning team at NASA Ames Research Center (ARC) and the University of Colorado/Boulder used satellite imagery of a lunar analog test site (Figure 1) at a resolution comparable to what is currently available for the Moon (approximately $0.5 \mathrm{~m} / \mathrm{pixel}$ ) and a derived digital elevation map to select a nominal site for the telescope deployment. In addition, the planning team created a set of rover task sequences to scout and survey the site, looking for potential hazards and obstacles to deployment.

\section{B. Phase 1: Site survey}

On June 17, 2013, Chris Cassidy (Figure 2, top), an astronaut on the ISS, remotely operated the NASA Ames K10 planetary rover for two hours to survey the test site from surface level. The survey data that was collected with K10 enabled identification of surface characteristics such as terrain obstacles, slopes, and undulations that are either below the resolution, or ambiguous due to the nadir pointing orientation, of orbital instruments. The mission planning team then analyzed the data and developed the final rover task sequences for telescope deployment.

\section{Phase 2: Payload deployment}

During the second test session on July 26, 2013, European Space Agency Astronaut Luca Parmitano (Figure 2, middle) remotely operated K10 for just over two hours to deploy three polyimide film-based antenna arms of a simulated telescope array. Parmitano first executed each deployment task sequence with the deployment device disabled, to

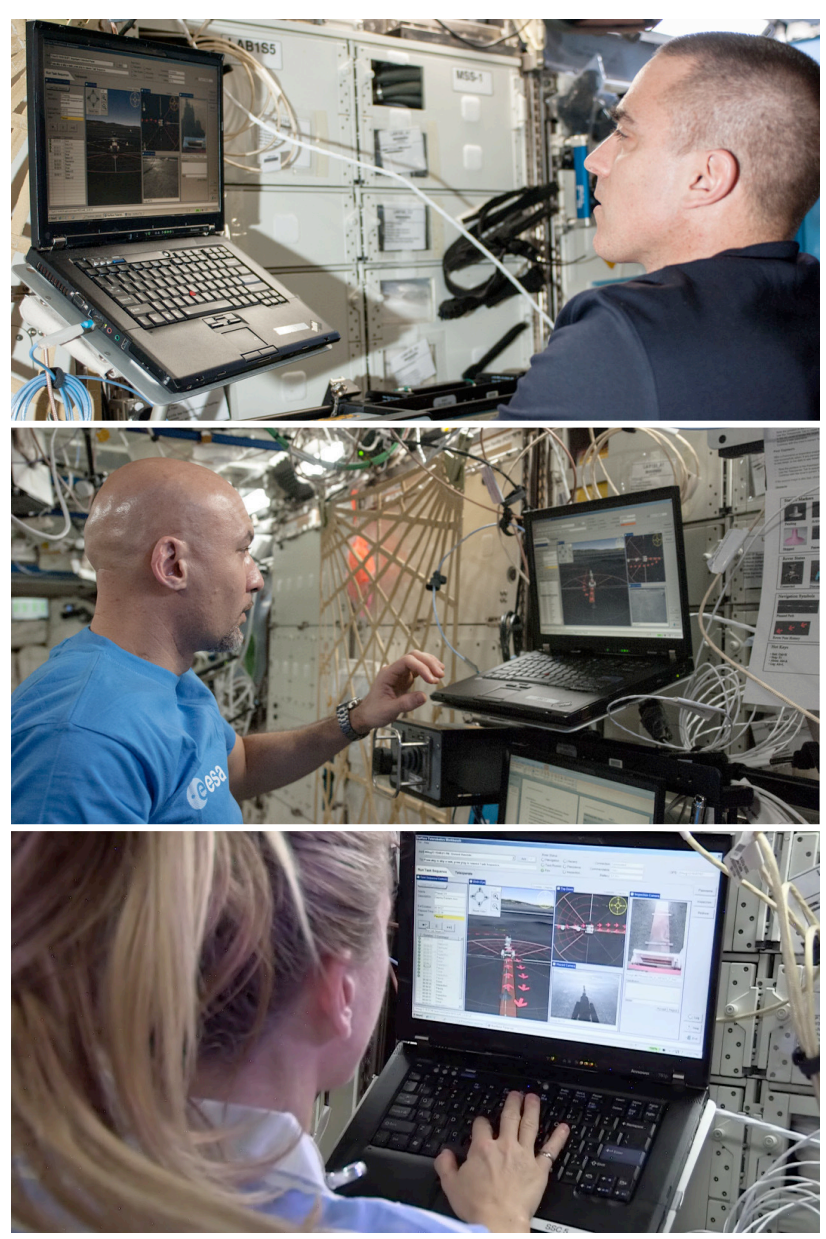

Figure 2. Astronauts Chris Cassidy (top), Luca Parmitano (middle), and Karen Nyberg (bottom) remotely operate the K10 rover from the ISS.

verify that the sequence is feasible. A high resolution, downward pointing camera focused on the film to document and to enable the astronaut to observe the deployment. Then, during actual deployment, Parmitano monitored both rover driving and antenna arm deployment. After the session was finished, the mission planning team reviewed deployment imagery and developed a set of rover inspection plans.

\section{Phase 3: Payload inspection}

During the final test session on August 20, 2013, NASA Astronaut Karen Nyberg (Figure 2, bottom) remotely operated K10 to perform detailed visual inspection of the deployed telescope. The primary objective for this phase was to obtain oblique, high-resolution camera views to document the deployed polyimide film antenna arms. A secondary objective was to search for possible flaws (e.g., folds and tears) in the material. Based on the inspection data, the mission planning team was then able to determine whether it would be necessary to repair, or replace, sections of the telescope array. 


\section{System Description}

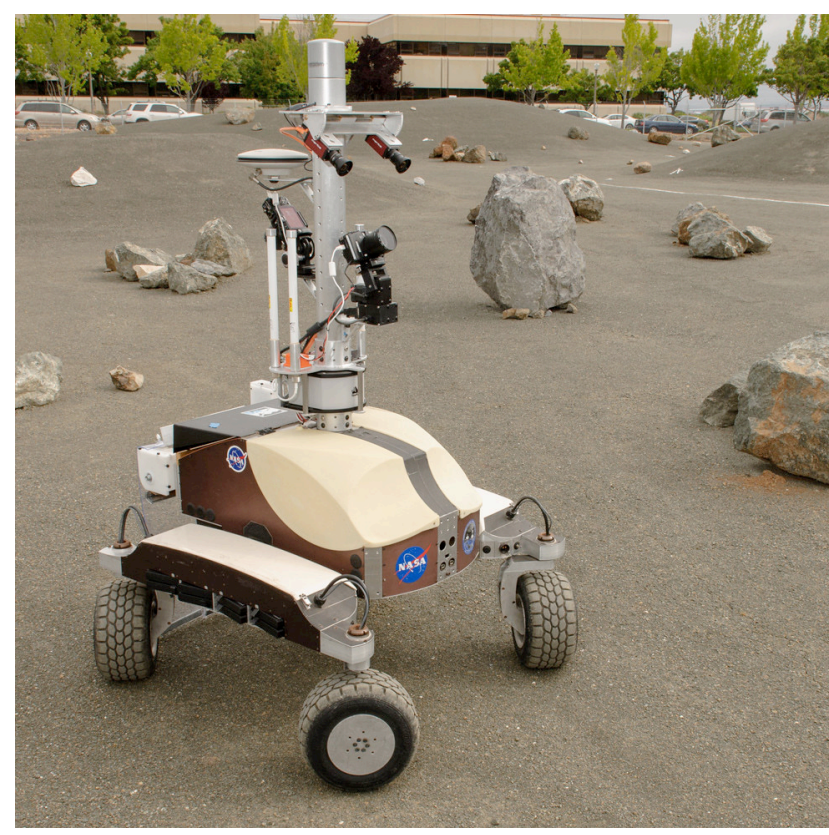

Figure 3. K10 planetary rover "Red" is equipped with a variety of sensors and instruments.

\section{A. K10 Planetary Rover}

The NASA Ames K10 planetary rover is shown in Figure 3. K10 has four-wheel drive, all-wheel steering and a passive averaging suspension. The suspension design helps balance wheel/soil forces and reduces the transmission of motion induced by travel over uneven ground. K10 is capable of fully autonomous operation on moderately rough natural terrain at human walking speeds (up to $90 \mathrm{~cm} / \mathrm{s}$ ).

K10's standard sensors include a Novatel differential GPS system and inertial measurement unit, a Honeywell digital compass, Point Grey Research IEEE 1394 stereo cameras, a Velodyne 3D scanning lidar, an Xsens inertial measurement unit, a suntracker, and wheel encoders.

K10's avionics design is based on commercial components. The robot is powered by twenty-four hot-swappable Inspired Energy 14.4V, 6.6 AH Li-Ion smart battery packs. K10's controller runs on a Linux-based laptop and communicates via a Tropos 802.11g mesh wireless system.

The K10 controller is based on our ServiceOriented Robotic Architecture (SORA). ${ }^{8}$ Major services include locomotion, localization, navigation, and instrument control. SORA uses high-performance middleware to connect services. Dependencies between services are resolved at service start. This approach allows us to group services into dynamic libraries that can be loaded and configured at run-time.

\section{B. Science Instruments}

To perform survey and inspection, we equipped the K10 rover with a panoramic camera and an inspection camera. Both instruments can provide contextual and targeted high-resolution color imaging of sunlit areas. These instruments are used for both science observations and situation awareness during operations.

The panoramic camera is a consumer-grade, 12 megapixel, digital camera (Canon PowerShot G9 camera) on a pan-tilt unit. We operate the camera at $350 \mathrm{rad} / \mathrm{pixel}$, which is comparable to the Mars Exploration Rover Pancam (280 rad/pixel). K10's panoramic camera, however, can be reconfigured for different resolutions by changing zoom. Images are mosaiced in software to create wide-field panoramic views.

The inspection camera uses the same camera model as the panoramic camera. However, the inspection camera is attached to K10 with a fixed rearpointing mount. The inspection camera is used to observe telescope film deployment as well as to perform inspection.

\section{Film Deployer}

Together with the University of Idaho, we developed and integrated a rear-mounted polyimide film deployer for the K10 rover (Figure 4). The deployer spools out $60 \mathrm{~cm}$-wide polyimide film, as a proxy for a lunar radio antenna, while the rover traverses planned deployment paths On-board software controls deployment: starting, stopping, and adjusting the tension on the film. For purposes of these tests, the film does not contain antenna or transmission line traces.

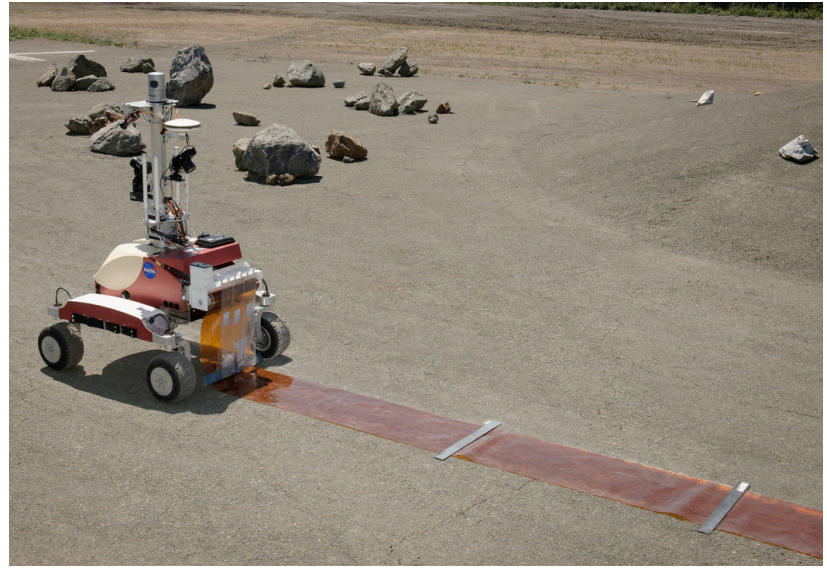

Figure 4. K10 deploys polyimide film to simulate deployment of a polyimide-based lunar radio telescope.

\section{User Interface}


The "Surface Telerobotics Workbench" (Figure 5 ) is used by ISS crew to remotely operate K10. The Workbench runs on a Space Station Computer is based on the "Visual Environment for Robotic Virtual Exploration" (VERVE), which is an interactive, 3D user interface for visualizing highfidelity $3 \mathrm{D}$ views of rover state, position, and task sequence status on a terrain map in real-time. ${ }^{9}$ VERVE also provides status displays of rover systems, renders 3D sensor data, and can monitor robot cameras. VERVE runs within the NASA Ensemble framework (based on the Eclipse RCP) and supports a variety of robot middleware, including the NASA Robot Application Programming Interface Delegate (RAPID). ${ }^{10}$

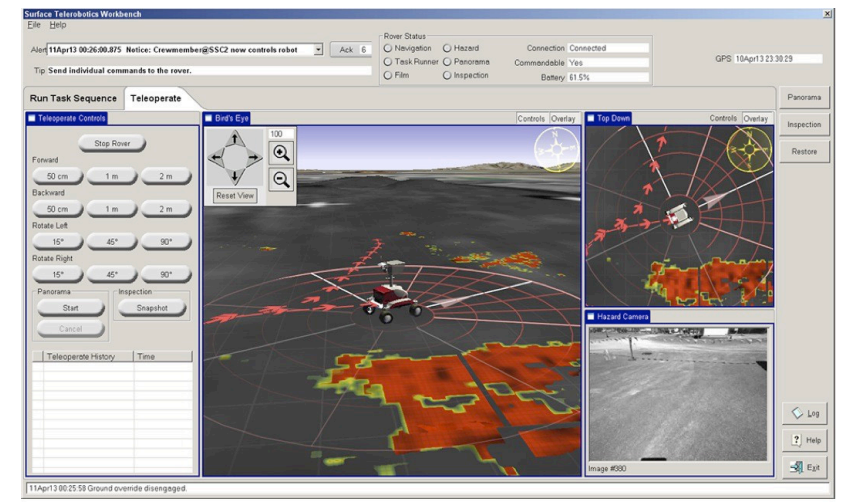

Figure 5. The "Visual Environment for Robotic Virtual Exploration" (VERVE) is an interactive 3D user interface for robot operations.

\section{E. Communications}

Figure 6 shows a simplified communications diagram for the Surface Telerobotics tests. Voice communications were carried over standard ISS and Payload Operations voice loops. The Rover Operations Lead at ARC communicates with the Payload Operations Director (POD) and Payload Communications Manager (PAYCOM) over the POD loop. Information for crew is relayed through PAYCOM over Space To Ground. Internal communications among the Rover Team - Operations, Science, Engineering, Logistics, Proxy, and Plug-in Port Utilization Officer (PLUTO) Support - occurres over the SS Coord voice loop.

Rover telemetry and commanding between the K10 rover and a laptop on the ISS is carried through a secure network data connection between the rover and the ISS Mission Control Center (MCC), which uses a proxy server machine on the MCC network. From there, traffic between the ISS laptop and the proxy server uses standard ISS Ku-band data communications through TDRSS (Tracking and Data Relay Satellite System).

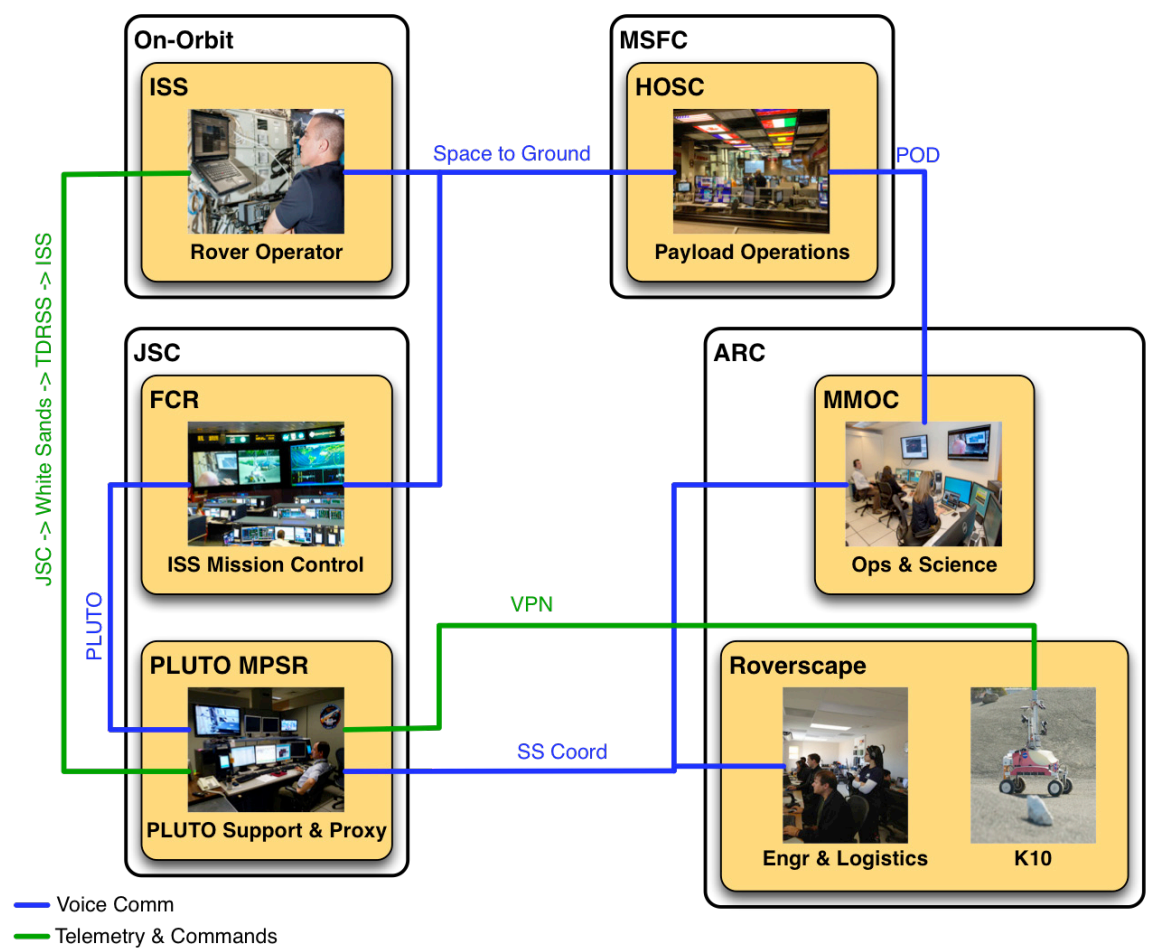

Figure 6. Voice and telemetry communications links. Voice loops are shown in blue. Telemetry (data) channels shown in green. 


\section{Assessment Approach}

\section{A. Characterize the Concept of Operations}

A key objective of the Surface Telerobotics tests was to characterize a concept of operations (or "conops") for a single crew member supervising a remote planetary rover with limited support from ground control on Earth. The concept of operations involves the following:

- The primary operations mode is supervisory control: task sequencing with interactive monitoring.

- The secondary operations mode is manual control (teleoperation): discrete commanding (relative position motions, direct instrument commands)

- Ground control handles major contingencies and strategic planning.

- The crew is responsible for tactical execution and modifications (minor deviations from the strategic plan) to handle minor contingencies and to achieve secondary mission objectives.

By characterizing this conops, we expected to better understand mission design and architectures, including mission timelining, mission duration and tempo, and how to best interleave different mission phases.

\section{Background}

To characterize the conops, we assessed the crew's situation awareness (SA) at all three stages of Endsley's model of SA formation ${ }^{11}$ :

- Level $1 \mathrm{SA}$ (Perception): What is the status, attributes, and dynamics of the elements relating to the environment, system, people, etc.?

- Level 2 SA (Comprehension): What is the impact of the perceptions?

- Level 3 SA (Projection): How are future states affected?

We also considered the five awareness categories (LASSO) ${ }^{12}$ used in the Urban Search and Rescue domain to help assess the operator's understanding of the robot. These aid in understanding what types of information crew uses at each level of SA.

- Location awareness

- Activity awareness

- Surroundings awareness

- Status awareness

- Overall mission awareness

To collect information about the crew's SA, we employed SAGAT ${ }^{13}$ questionnaires. We also used the Bedford Workload Scale ${ }^{14}$ to obtain subjective assessment of crew workload.

\section{SAGAT}

SAGAT queries were presented to crew at random times throughout each test session on a secondary laptop. Crew was required to look away from the primary laptop that hosted the rover user interface while answering SAGAT questions.

Development of the SAGAT queries is based on a high level goal-directed task analysis in order to understand what aspects of the situation contribute to crew's SA. The analysis was cross-referenced with the LASSO categories to understand what aspects of the robot crew must understand.

Questions were then formulated based on the decisions we expected crew to make and referenced against the types of information provided to crew at all times through the user interface. The terminology used was the same as that used in the training materials provided to crew. The list of questions was cross-referenced with the SA Levels and LASSO categories.

\section{B. Characterize the System}

Another objective of these tests was to characterize system utilization and performance for a single crewmember supervising a remote planetary rover with limited backup from ground control. We then collected a variety of engineering data (rover position, power levels, health, data messages, etc.) to assess what is needed to make such a system work.

Our system is defined by the following:

- Robot: ARC K10 rover with the following instruments:

$\circ$ panoramic color camera system (used for survey and inspection) 
- forward-facing monochromatic stereo cameras (used for driving)

- single rear-facing camera (used for antenna film deployment monitoring and inspection)

- User interfaces

- Surface Telerobotics Workbench rover monitoring and task sequence editing tool for use by both the ground team and crew

- Middleware

○ RAPID

\section{Metrics}

The rover system, including remote supervisory tools, can be characterized by computing metrics for mission success, robot asset utilization, task sequence success, system problems, and robot performance. For this objective we rely on metrics based on rover telemetry monitoring supplemented by human observations made during the evaluation. We can compute metrics using logs of rover telemetry recorded during each test session. Data from these logs can also be played back through performance monitoring software that encodes metrics algorithms and saves metrics values to file.

The Surface Telerobotics sessions simulated operations that include some anomalies for the astronaut to handle. These anomalies included: (1) obstacles in the rover's path that required the astronaut to teleoperate the rover around them, (2) poor quality images that required the astronaut to retake the image, and (3) low power levels that required the astronaut to abandon nominal rover activities and discuss the issue with ground control. This knowledge of the planned activities and expected anomalies can be used to define the expected values for the mission "as planned".

The metrics that characterize the system based on rover telemetry are described below.

\section{Mission Success Metrics}

Mission Success metrics indicate whether rover task sequences complete successfully and have the intended effect. Examples of questions answered by metrics for mission success include:

- What percentage of the task sequences: (a) completed normally, (b) ended abnormally (failed tasks, aborted tasks, tasks not attempted), or (c) were not attempted?

- What percentage of the task sequences was scheduled and what percentage was unscheduled (i.e., in reaction to anomalies)?

Using these metrics we can determine whether task sequences ended normally or not, the percentage of task sequences that were scheduled and unscheduled, if scheduled or unscheduled activities ended abnormally, whether all data were collected, and if the telescope arms were deployed as planned. To identify if certain types of task sequences are causing the astronaut more difficulty, abnormal task sequence completion and the number of task sequence repeats is tracked by type of task sequence.

\section{Robot Asset Utilization Metrics}

Robot Asset Utilization characterizes how the robot system was used, and whether this utilization contributed to mission success. Examples of questions answered by metrics for robot asset utilization include:

- What percentage of the time did the robot spend on different types of tasks (traverse, panoramic imaging, inspection imaging)? How did actual time in task compare to the expected time?

- Did rover drive the expected distance?

Using these metrics we can determine how rover time was spent during the session, including how much time the rover was waiting or idle. We also can compare how much time was spent doing different types of tasks relative the expected time on these tasks. Assuming we can make reasonable estimates of time for different tasks, this should help identify types of tasks where problems occurred.

\section{Task Success Metrics}

Task Success metrics characterize the individual tasks performed by the rover, such as drive to a waypoint, or take an image. Metrics for task success are similar to the mission success metrics, except they are computed for individual tasks in the task sequence instead of looking at the task sequence as a whole. Example questions answered by metrics for task success include:

- What percentage of the tasks: (a) ended normally, (b) ended abnormally, or (c) were not attempted? Show this per session and per task sequence. 
- What percentage of the tasks that ended abnormally was in scheduled task sequences, and what percentage was in unscheduled task sequences?

Using these metrics we can determine whether tasks ended normally or not, the percentage of tasks that were scheduled and unscheduled, and if scheduled or unscheduled tasks ended abnormally. To identify if certain types of tasks caused the astronaut difficulty, we could track the number of task re-tries and whether retried tasks are successful.

\section{System Problem Metrics}

System Problem metrics identify what system anomalies were observed and how much time was spent in handling them. One observable indicator of a system problem is the detection of a Caution and Warning (C\&W) state. For K10, this includes joint errors, subsystem errors, and planner failures. Given that the robot was remotely operated in the controlled environment of the ARC test facility, not many of these errors occured. Consequently, our approach is to measure the number of $\mathrm{C} \& \mathrm{~W}$ states that occur for each session and the time spent handling them, but we do not consider the time spent handling these problems as human intervention (within the simulation). The following rover $\mathrm{C} \& \mathrm{~W}$ states are considered:

- Emergency stop

- Position error

- Steering warning and error

- Over current

- Navigator or locomotor subsystem failure

- Panoramic camera subsystem failure

- Inspection camera subsystem failure

- Mission subsystem failure

For these sessions, unscheduled human intervention occured in response to contingencies. Contingencies that the astronaut was trained to handle include re-taking an image, moving the rover around an obstacle in the path, and redirecting the rover activities if power levels dropped too low. We can measure the Mean Time To Intervene (MTTI) as the mean time spent executing unscheduled task sequences, and Mean Time Between Interventions (MTBI) as the mean time between unscheduled task sequences ${ }^{15}$. Together MTTI and MTBI indicate the portion of the session spent by the astronaut handling rover contingencies.

\section{Robot Performance Metrics}

Robot Performance metrics characterize how well the robot performs assigned tasks. We measure the timeliness of robot task performance as the ratio of the actual time to execute a task sequence or task to the expected time to execute a task sequence or task. The usefulness of these measures depends on the ability to obtain good task sequence duration estimates. Metrics for robot performance answer the following questions:

- Did the rover take the expected time to execute task sequences? How many task sequences took longer than expected? How much longer?

- Did the rover take the expected time to execute tasks? How many tasks took longer than expected? How much longer?

Measures of mission success and task success indicating abnormal performance also can help characterize robot task performance. We could measure counterproductive motion of the robot (i.e., motion away from the target) as an indicator of traverse effectiveness. For these sessions, however, counterproductive motion was minimal, so we do not believe such measures would be particularly diagnostic.

\section{Preliminary Results}

Our initial analysis focused on characterizing robot utilization based on the K10 rover telemetry that we recorded during the Surface Telerobotics tests. We used a variety of metrics based on earlier work in computing human-robot performance in real-time. ${ }^{16}$ In this section we describe these performance metrics and report results for Session 1. We expect to perform similar analyses for Sessions 2 and 3 using telemetry recorded from the K10 rover. During Session 1 Astronaut Chris Cassidy completed all the task sequences of Phase 1 and continued on to five of seven sequences of Phase 2. 
Performance metrics are computed by (1) partitioning each phase into meaningful categories of work and rest (called wait periods), (2) detecting events that indicate transitions between these categories, and (3) aggregating the time spent in each category. The work and wait periods are defined such that only one category applies at any time.

Work Periods

- Execute: corresponds to all work done when a planned autonomous rover task is active. The astronaut may perform supervisory tasks in parallel with the rover, depending upon type of rover task.

- Teleops: corresponds to the work done when the astronaut manually tele-operates the rover.

- Idle_in_Plan: corresponds to work done by the astronaut in support of the rover's planned tasks. For example, the rover is paused while the astronaut inspects images taken during antenna deployment.

- Questionnaire: corresponds to work done by the astronaut answering questions assessing situation awareness and workload. During this work period the rover is paused.

\section{Wait Periods}

- Time_before_Start: corresponds to the time period after a task sequence is selected for execution but before the first task in the task sequence is executed.

- Wait_between_Plans: corresponds to the time period when the rover has no task sequence to perform.

- LOS: corresponds to the time period when all work is paused due to loss of communication signal.

- Time_in_Problem: corresponds to time when the rover is paused due to a problem.

All six Phase 1 task sequences were performed with no significant problems. Five panoramas were taken as expected, two of them with pointing contingencies where the image was taken in wrong direction. In one of the two panorama contingencies, the astronaut re-took the image from the correct direction. $16 \%$ of Phase 1 was spent with the astronaut tele-operating the robot out of planned rover trap contingencies.

Five of seven Phase 2 task sequences were completed normally. The sixth task sequence was paused partway through because the USB bus on the rover went down. Because of this problem, Session 1 was ended approximately 10 minutes early without completing task sequences 2.06 and 2.07. There were no tele-operations during Phase 2 because there were no rover trap contingencies in this phase. Phase 2 had a larger amount of time idle in the task sequence because the rover was paused while the crew reviewed the inspection images during antenna deployment.

The astronaut filled out 4 questionnaires in each phase. More time was spent answering questions in Phase 1 because problems with the questionnaire form required the astronaut to fill it out differently than originally instructed. Phase 1 has almost no time lost to LOS while $24 \%$ of Phase 2 was spent in LOS. Figure 7 (a) and (b) summarize work breakout for Phases 1 and 2, respectively
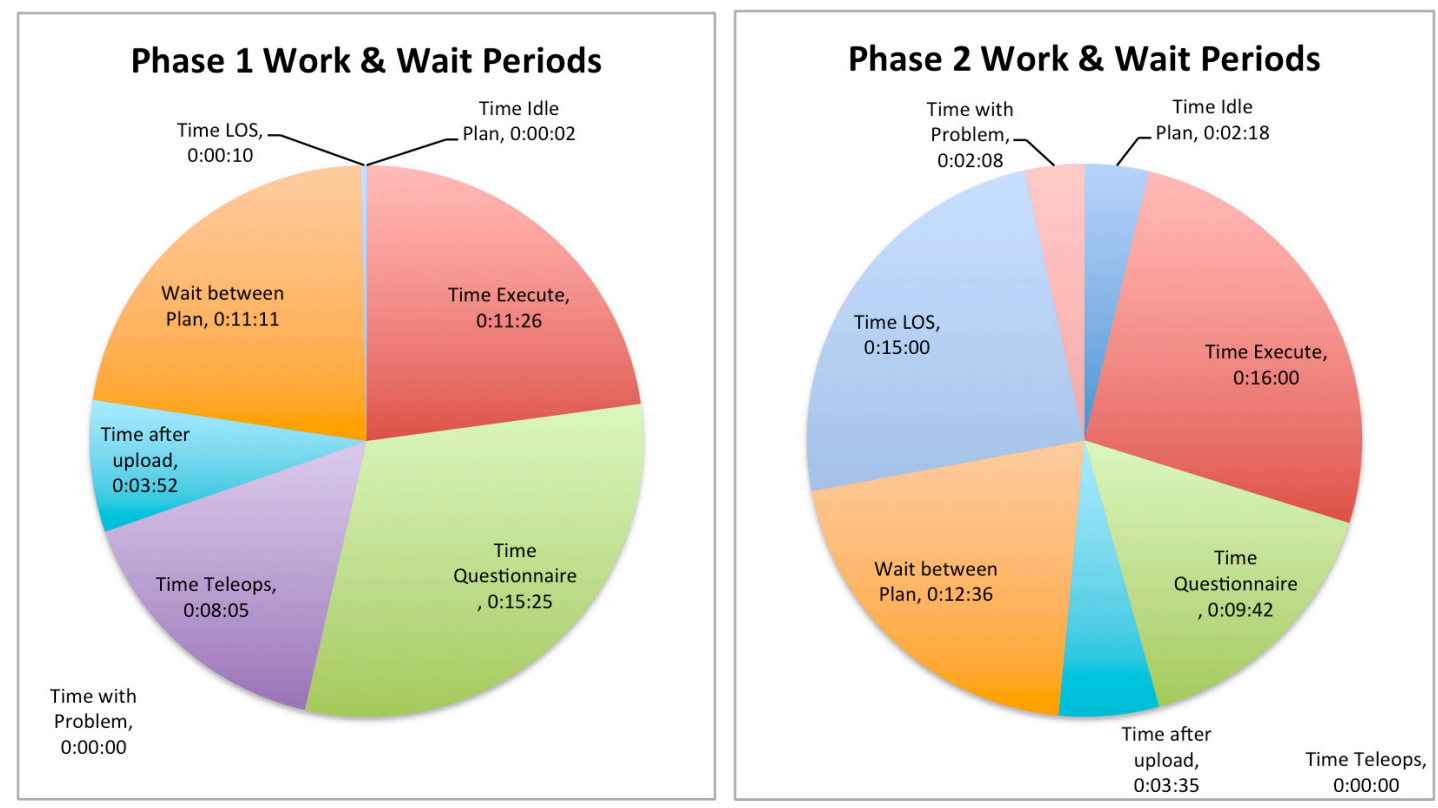

Figure 7. Work and Wait periods for Phase 1 (a) and Phase 2 (b) of Session 1, respectively. 
Productivity Measures: Productivity refers to the time during a phase when the astronaut and rover are performing tasks that contribute toward the mission objectives. For this experiment the four work periods described earlier are considered productive. Consequently productive time $(P T)$ for each phase is the sum of all work periods for that phase. Overhead time (OT) refers to the time during a phase when the astronaut and rover are not waiting, and is the sum of all wait periods for the phase. $\% P T$ is the percentage of the phase in productive time. \%OT is the percentage of the phase in overhead time. The ratio of PT to OT is called Work Efficiency Index.${ }^{17}$ For this analysis we remove Loss-of-Signal (LOS) time from the time in phase. Table 1 shows the productivity measures for Session 1. In summary the productivity $\% \mathrm{PT}$ of the human-robot team averaged $\sim 65 \%$ for Session 1 .

Table 1. Productivity measures for Session 1.

\begin{tabular}{|l|c|c|c|c|c|c|}
\hline Productivity & Total Time & PT & OT & \%PT & $\%$ OT & WEI \\
\hline Phase 1 & $0: 50: 01$ & $0: 34: 58$ & $0: 15: 03$ & 69.90 & 30.10 & 2.32 \\
\hline Phase 2 & $0: 46: 19$ & $0: 28: 00$ & $0: 18: 19$ & 60.45 & 39.55 & 1.53 \\
\hline
\end{tabular}

Distance Traveled Measures: Distance traveled is the total distance driven by the K10 rover during each task sequence, whether remotely driven with supervisory or manual control. In Session 1, the rover performed eleven task sequences, which covered a total distance of $221 \mathrm{~m}$.

Figure 8 shows distance traveled in each task sequence. The rover covered an average distance of $20 \mathrm{~m}$ per task sequence. The longest task sequence covered a distance of $49 \mathrm{~m}$. When K10 was operated using supervisory control, the rover drove at average speed of $40 \mathrm{~cm} / \mathrm{s}$. The average speed over the total duration of Session 1, which lasted $96 \mathrm{~min}$, was approximately $3.8 \mathrm{~cm} / \mathrm{s}$.

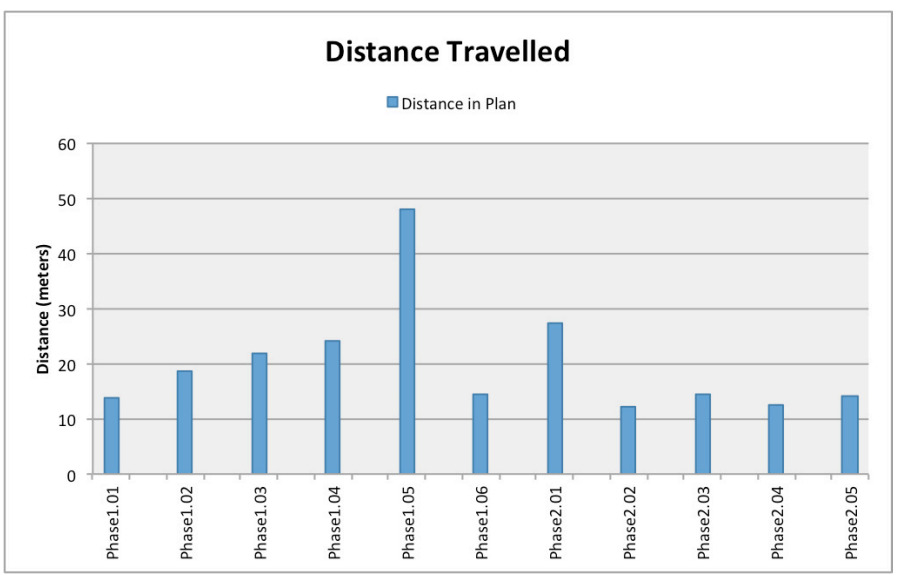

Figure 8. Distance traversed by the K10 rover during each task sequence of Session 1.

\section{Conclusion}

We developed the Surface Telerobots tests to study how astronauts in a flight vehicle can remotely operate a surface robot across a short time delay. We carried out three test sessions in Summer 2013 during ISS Increment 36 and collected a wide range of engineering data to improve our understanding of how to: (1) deploy a crew-controlled telerobotics system for performing surface activities and (2) conduct joint human-robot exploration operations.

Three ISS astronauts (Chris Cassisdy, Luca Parmitano, and Karen Nyberg) remotely operated the K10 rover for a combined total of approximately $10.5 \mathrm{hr}$ to simulate a proposed, future lunar exploration mission. The astronauts used a combination of supervisory control (task sequencing) and teleoperation (discrete commanding) and to remotely operate K10 in an outdoor test area at the NASA Ames Research Center. The astronauts monitored the rover interactively, with only minimal $(500-750 \mathrm{msec})$ communications latency and intermittent LOS periods.

Preliminary data analysis suggest that the technologies developed for analog ground simulations of remote supervisory control of a planetary rover remain highly effective when used on-orbit. In addition it appears that (1) rover autonomy, particularly hazard detection and safeguarding, greatly enhanced operational efficiency and robot utilization; (2) interactive 3-D visualization of robot state and activity reduced operator workload and increased situation awareness; and (3) command sequencing with interactive monitoring was a highly effective strategy for crew-centric surface telerobotics.

We plan to perform detailed data analysis during Fall 2013, with emphasis on characterizing the use and performance of rover software, crew user interfaces, and operations protocols. The results and lessons learned from Surface Telerobotics will be used to further mature technologies required for future deep-space human missions, including robot planning and commanding interfaces, automated summarization and notification systems for situation awareness, on-board robot autonomy software, data messaging, and short time-delay mitigation tools. 


\section{Acknowledgments}

First and foremost, we would like to thank Jack Burns, Laura Kruger, and the Lunar University Network for Astrophysics Research (LUNAR) for developing the Orion MPCV L2-Farside mission concept and for their support of Surface Telerobotics. We also thank Josh Hopkins, William Pratt, and Chris Norman of Lockheed Martin Corporation for insightful discussions on the Orion MPCV. Sophie Milam and George Korbel of the University of Idaho developed the polyimide film deployer for K10. Industrial design students from the Academy of Art University in San Francisco collaborated to create the Surface Telerobotics Workbench.

We would like to acknowledge the dedication and tireless effort of crew office (particularly Chris Cassidy, Luca Parmitano, and Karen Nyberg), the JSC Mission Operations Directorate (particularly Mike Halverson), the NASA Lunar Science Institute, the ISS Tech Demonstration office, ISS Avionics and Software and NASA public affairs (particularly Rachel Hoover, Dave Steitz, and Maria Quon). Brett Beutter, Don Kalar, Josh Hopkins, William Pratt and Chris Norman all served as simulated crew during operational readiness tests.

We especially thank Bonnie James, Randy Lillard, the NASA Technology Demonstration Missions Program office, Jason Crusan, and Chris Moore for their continued advocacy and support. The NASA Technology Demonstration Missions Program (NASA Space Technology Mission Directorate) provided funding for this work.

This paper is dedicated to the memory of Astronaut Janice Voss, who helped plan Surface Telerobotics and who served as the initial crew office liason for the project.

\section{References}

1 Augustine, N., et al., "Seeking a Human Spaceflight Program Worthy of a Great Nation," Review of U.S. Human Spaceflight Plans Committee, Doc No. PREX 23.2:SP 1/2, 2009.

2 Hopkins, J., "Stepping Stones: Exploring Increasingly Challenging Destinations on the Way to Mars", briefing to Human Spaceflight Architecture Team, Lockheed Martin.

3 Korsmeyer, D., Landis, R., et al. "A Flexible Path for Human and Robotic Space Exploration”, 2010 AIAA Space Operations Conference, AIAA, Washington, DC, 2010.

4 NASA, “Consolidated Destinations Cycle B Briefing,” Human Spaceflight Architecture Team, July 12, 2011.

5 Nergaard, K., de Frescheville, F. B., et al., “METERON CDF Study Report: CDF-96(A)” European Space Agency, 2009.

6 Burns, J. O., et al, “A Lunar L2-Farside Exploration and Science Mission Concept with the Orion Multi-Purpose Crew Vehicle and a Teleoperated Lander/Rover," Advances in Space Research 52, 2013.

7 Committee for a Decadal Survey of Astronomy and Astrophysics, 2010, National Research Council, New Worlds, New Horizons in Astronomy and Astrophysics, The National Academies Press, Washington, DC, 2010.

8 Flückiger, L., and Utz, H., "Field tested service oriented robotic architecture: Case study," International Symposium on Artificial Intelligence, Robotics, and Automation in Space (iSAIRAS), 2012.

9 Lee, S. Y., et al, "Reusable science tools for analog exploration missions: xGDS Web Tools, VERVE, and Gigapan Voyage," Acta Astronautica, Vol. 90, No. 2, October 2013, pp. 268-288.

${ }^{10}$ Torres, R. J., Allan, M., Hirsh, R., Wallick, M.N., "RAPID: Collaboration results from three NASA centers in commanding/monitoring lunar assets," IEEE Aerospace Conference, IEEE, 2009.

${ }^{11}$ Endsley, M. R, "Measurement of Situation Awareness in Dynamic Systems, Human Factors," The Journal of the Human Factors and Ergonomics Society, Vol. 37, No. 1, March 1995, pp. 65-84.

12 Drury, J. L., Keyes, B., Yanco, H. A., "LASSOing HRI: analyzing situation awareness in map-centric and video-centric interfaces," 2nd ACM/IEEE International Conference on Human-Robot Interaction (HRI), IEEE, 2007.

${ }^{13}$ Endsley, M. R., and Garland, D. J., eds, Situation awareness analysis and measurement, CRC Press, Boca Raton, FL, 2000.

${ }^{14}$ Roscoe, A.H., and Ellis, G.A., "A subjective rating scale assessing pilot workload in flight. A decade of practical use," Royal Aerospace Establishment, Technical Report 90019. Farnborough, UK: Royal Aerospace Establishment, 1990.

${ }^{15}$ Arnold, J., "Towards a framework for archi-tecting heterogeneous teams of humans and robots for space exploration", M.S. Thesis, Dept. of Aeronautics and Astronautics, Massachusetts Institute of Technology, Cambridge, MA, 2006.

${ }^{16}$ Schreckenghost, D., Fong, T., and Milam, T., "Measuring Performance in Real-time during Remote Human-Robot Operations with Adjustable Autonomy," IEEE Intelligent Systems, Vol. 25, No. 5, Sept./Oct. 2010, pp. 36-45.

${ }^{17}$ Gernhardt, M., “Work Efficiency Indices,” Presentation at Johnson Space Center, November 15, 2005. 